haemoglobin prior to endoscopy was $92 \mathrm{~g} / \mathrm{L}$. $40 \%$ were on anticoagulants or anti-platelets on admission. Of all patients who needed Hemospray, 43 had gastroscopies, 1 ERCP and 1 had colonoscopy. The details of diagnosis are in table 1 .

- $23.39(86 \%)$ patients had Hemospray as adjunct therapy \& all patients had complete haemostasis documented at the end of index procedure.

- 24. It was used as the only modality of therapy successfully in 6 patients (4 with malignancy and 2 iatrogenic bleeds)

- 25. Overall, 6/45 patients had a documented rebleed after discharge but within 4 weeks; 4 had bleeding vessels and were on warfarin or Ticagrelor; 2 patients had tumour related recurrent bleeding.

- 26. Forrest classification applicable to 22 patients $-1 \mathrm{~A}(\mathrm{n}=3$, $15 \%), 1 \mathrm{~B}(\mathrm{n}=10,46 \%), 2 \mathrm{~A}(\mathrm{n}=3,15 \%), 2 \mathrm{~B}(\mathrm{n}=3,15 \%), 2 \mathrm{C}$ $(\mathrm{n}=2,9 \%)$.

- $27.100 \%(n=7)$ of UGIB related to malignancy responded to Hemospray (adjunct and monotherapy included)

- 28. 30 day mortality was $24 \%(n=11)$ with 3 deaths directly attributed to GI bleeding (4 palliative secondary to malignancy and 4 multifactorial in elderly patients).

Conclusions Our 4 year experience suggests that Hemospray can be a useful adjunct to conventional endoscopic therapy for acute GI bleed with success rates comparable to previous reports (nearly $85 \%$ in our cohort), with a rebleed rate of $14 \%$. Our findings suggest it could be useful as monotherapy for bleeding related to malignancies where other therapy may be impractical. More studies with larger numbers are needed to add to the evidence base.

\begin{tabular}{ll} 
Abstract PTH-056 Table 1 & \\
\hline Diagnosis & Number $\mathbf{n}=45$ \\
\hline Duodenal Ulcer & $14(32 \%)$ \\
Oesophageal/gastric ulcer & $4(9 \%)$ \\
Malignancy related & $7(15 \%)$ \\
latrogenic bleeding & $7(15 \%)$ \\
Other including unclear cause of bleed & $13(29)$ \\
\hline
\end{tabular}

\section{PTH-057 ENDOSCOPIC MANAGEMENT OF BURIED BUMPER SYNDROME}

Angad Singh*, Andrea Cartwright, Rizwan Kassam, Javaid Subhani. Basildon University Hospital, Basildon, UK

\subsection{6/gutjnl-2018-BSGAbstracts.78}

Introduction Buried Bumper Syndrome (BBS) is an uncommon, yet potentially serious complication of percutaneous gastrostomy tube (PEG) placement with a quoted incidence of $1 \%$. Usually identified during routine nursing assessment, release of a buried bumper can be achieved via external manipulation, endoscopically or via surgery.

Aim Review the frequency and success of endoscopic treatment of BBS.

Methods At Basildon University Hospital a 4-level stepwise approach was adopted for management of Buried Bumper Syndrome, with each level representing an escalation of therapy.
- 29. Level 1- Release achieved by external manipulations by a nurse or radiologist.

- 30. Level 2- Endoscopic if the internal bumper can be seen through a relatively wide tract it may be pulled through by graspers or snare.

- 31. Level 3-Sometime the tract is narrow and may require quadrantic incisions ( $3 a$ ) or subsequent balloon dilations ( $3 b$ ) before release.

- 32. Level 4- Cannot be endoscopically released and require surgery.

Retrospective review of endoscopy records held on Unisoft from August 2009 to Jan 2018 by a bespoke Access query. Endoscopy and clinical records were reviewed of the identified patients. By definition no Level 1 cases would be identified.

Results 27 incidences of BBS requiring 30 endoscopic procedures in 18 patients were identified. 4 (22\%) patients had repeated distinct episodes of BBS (2-5). 18 episodes of BBS were successful resolved endoscopically (67\%). Interventions were 4x Level 2, 9x level 3a, 5x Level 3b.

Endoscopic treatment of 9 episodes of BBS were unsuccessful (Level 4). 4/9 patients had a surgical removal, though one precipitated a 2 month admission complicated by an ITU stay. The other 5 patients were deemed unfit, though one patient continued to suffer from septic episodes related to the bumper.

The PEG insertion date was unknown many patients (e.g out of area, pre-2009) but for 12 patients the time from insertion to attempted release of BBS was known. 2 episodes $<12$ months, 3 occurred at 12 months, 3 at $21-25$ months, 4 at $31-33$ months. The only short (2 months) interval was in a patient with previous PEG.

Conclusion This is a relatively infrequent endoscopic dilemma with only 27 instances over a 8.5 year study period. However with rare scenarios the referral pathway can be unclear. Only one endoscopist managed Level 3 releases and by default they became the routine destination for Nutrition team referrals. This allowed a $66 \%$ success rate but disappointingly $22 \%$ of the patients had recurrent episodes. Recommendations 1) Education of the institutions caring for these patients. 2) Single endoscopists to develop expertise in each centre 3) If successful, attempt to site a new PEG in a second area despite the difficulty.

\section{PTH-058 SMART COLONOSCOPY:USING BIG-DATA TO IDENTIFY PREDICTORS OF NORMAL COLONOSCOPIC EXAMINATIONS}

Matt Stammers, Sreedhari Thalasekaran, Pradeep Bhandari. Queen Alexandra Hospital, Portsmouth, UK

\subsection{6/gutjnl-2018-BSGAbstracts.79}

Introduction Endoscopy workload is increasing at a faster pace than available resources. The NHS has a wealth of data, which if used properly can improve resource allocation in future.

The aim of this study was to review mass colonoscopy data to identify those factors most associated with a normal examination, in order to help rationalise future resource utilisation.

Methods We constructed a standardised, anonymised database, containing all colonoscopies performed locally between 01/01/ 